\title{
Prova de Trabalho de Parto Após uma Cesárea Anterior
}

\author{
The Trial of Labor After one Cesarean Section \\ Iracema de Mattos Paranhos Calderon ${ }^{1}$, Jacqueline Leite Frade ${ }^{1}$, Joelcio Francisco Abbade ${ }^{1}$ \\ Carolina Prado Diniz ${ }^{1 *}$, Ivete Dalben ${ }^{2}$, Marilza Vieira Cunha Rudge ${ }^{1}$
}

\section{RESUMO}

Objetivo: estudar a validade da prova de trabalho de parto (PTP) em gestantes com uma cesárea anterior.

Métodos: estudo retrospectivo, tipo coorte, incluindo 438 gestantes com uma cesárea anterior ao parto em estudo e seus 450 recém-nascidos (RN), divididas em dois grupos - com e sem PTP. O tamanho amostral mínimo foi de 121 gestantes/grupo. Considerou-se variável independente a PTP e as dependentes relacionaram-se à ocorrência de parto vaginal e à freqüência de complicações maternas e perinatais. Foram efetuadas análises uni $e$ multivariada, respectivamente. A comparação entre as freqüências (\%) foi analisada pelo teste do qui-quadrado $\left(\chi^{2}\right)$ com significância de $5 \%$ e regressão logística com cálculo do odds ratio (OR) e do intervalo de confiança a $95 \%$ (IC $\left.{ }_{95 \%}\right)$.

Resultados: a PTP associou-se a 59,2\% de partos vaginais. Foi menos indicada nas gestantes com mais de 40 anos (2,7\% vs 6,5\%) e nas portadoras de doenças associadas e complicações da gravidez: sindromes hipertensivas $(7,0 \%)$ e hemorragias de $3^{\circ}$ trimestre $(0,3 \%)$. A PTP não se relacionou às complicações maternas e perinatais. As gestantes que tiveram o parto por cesárea, independente da PTP, apresentaram maior risco de complicações puerperais $(O R=$ 3,53; $\left.I C_{95 \%}=1,57-7,93\right)$. A taxa de mortalidade perinatal foi dependente do peso do RN e das malformações fetais e não se relacionou à PTP. Ao contrário, as complicações respiratórias foram mais freqüentes nos $R N$ de mães não testadas quanto à $P T P\left(O R=1,92 ; I C_{95 \%}=1,20\right.$ 3,07).

Conclusões: os resultados comprovaram que a PTP em gestantes com uma cesárea anterior é estratégia segura - favoreceu o parto vaginal em 59,2\% dos casos e não interferiu com a morbimortalidade materna e perinatal. Portanto, é recurso que deve ser estimulado.

PALAVRAS-CHAVE: Prova de trabalho de parto. Cesárea prévia. Complicações do parto. Mortalidade perinatal. Cesárea.

\section{Introdução}

O aumento alarmante e crescente das taxas de cesárea é realidade mundial, representando problema de saúde pública, por se associar a morbidade materna e fetal e a elevados custos hospitalares ${ }^{1}$. No Brasil adquire caráter epidêmico, com conseqüente aumento da mortalidade materna ${ }^{2}$, exigindo medidas eficazes para a reso-

\footnotetext{
${ }^{1}$ Departamento de Ginecologia e Obstetrícia

2 Departamento de Saúde Pública da Faculdade de Medicina de Botucatu - UNESP

* Bolsista Iniciação Científica - PIBIC/CNPq

Correspondência:

Iracema de Mattos Paranhos Calderon

Rua Atílio Losi, 226 - Jardim Paraíso

18610-260 - Botucatu - SP

e-mail: calderon@fmb.unesp.br
}

lução do problema.

A cesárea predispõe a diversas complicações, tais como: infecção puerperal, gravidez ectópica, histerectomia intraparto ou puerperal, acidentes tromboembólicos, má implantação placentária na gestação posterior (placenta prévia e abruptio placentae) e distúrbio respiratório no recém-nascido (RN), além da prematuridade iatrogênica ${ }^{3}$.

O Colégio Americano de Ginecologia e Obstetrícia (ACOG) recomenda a prova de trabalho de parto (PTP) em gestantes com uma cesárea anterior $^{4}$, com índices de sucesso de 60 a $90 \%{ }^{3-5}$. Entretanto, nas últimas duas décadas a cesárea transformou-se em recurso freqüente para resolução da gestação, principalmente em gestantes com cesárea prévia, influenciando no crescimento destes indices ${ }^{2}$. 
A PTP é definida por contrações uterinas, espontâneas ou induzidas artificialmente, rítmicas e progressivas, objetivando o parto vaginal. É contra-indicada nos casos de cicatriz uterina não segmentar, antecedente de rotura uterina, desproporção céfalo-pélvica absoluta, placenta prévia e apresentação anômala ${ }^{3}$.

Vários autores têm testado indicadores de sucesso para esta prova, destacando-se o índice de Bishop favorável, a ocorrência de parto vaginal posterior à cesárea e algumas indicações da cesárea prévia, tais como apresentação pélvica, préeclâmpsia, gravidez gemelar e placenta prévia ${ }^{6,7}$.

As complicações da PTP em gestantes com cesárea anterior são mínimas e dependentes do adequado acompanhamento médico, da vigilância do bem-estar fetal e do respeito às contra-indicações. A morbidade é duplicada na falha da PTP, pelo risco de rotura uterina, histerectomia e complicações operatórias ${ }^{3,8-11}$.

Rosen et al. ${ }^{9}$, em estudo de meta-análise, observaram maior índice de mortalidade nos RN de mães submetidas à PTP comparados aos nascidos por cesárea eletiva. No entanto, outros autores demonstraram que o índice de mortalidade perinatal das parturientes submetidas à PTP não se diferenciou do observado nas cesáreas eletivas ${ }^{10,11}$. Pires ${ }^{12}$ comprovou que a PTP em gestantes com uma cesárea anterior não modificou a condição de vitalidade do neonato, parecendo estar mais relacionada à vigilância do bem-estar fetal intraparto e à ocorrência de rotura uterina.

A literatura mundial recomenda a PTP em gestantes com uma cesárea anterior na viabilização do parto vaginal, sem aumentar as complicações maternas e perinatais. No Brasil, onde é premente a necessidade de conter os índices alarmantes de cesáreas desnecessárias, poucos estudos foram desenvolvidos ${ }^{12}$.

Este trabalho teve como objetivo geral estudar a validade da PTP na obtenção do parto vaginal em mulheres com uma cesárea anterior. Foram objetivos específicos determinar a ocorrência de parto vaginal e de complicações maternas e perinatais em gestantes submetidas à PTP.

\section{Pacientes e Métodos}

A pesquisa foi realizada na Maternidade do Hospital das Clínicas de Botucatu, no período de janeiro de 1996 a janeiro de 1998. Trata-se de um estudo retrospectivo, tipo coorte, sobre as práticas assistenciais relacionadas à PTP em gestantes com uma cesárea anterior. Foram incluídas 438 gestantes com uma cesárea anterior, representando $18 \%$ do total de 2.432 partos no período.

As gestantes foram divididas em dois grupos, de acordo com a realização ou não da PTP, espontânea ou induzida: PTP-Sim - parturientes com uma cesárea anterior submetidas à PTP, $\mathrm{n}$ = 299; PTP-Não - parturientes com uma cesárea anterior não submetidas à PTP, $\mathrm{n}=139$.

Para o cálculo do tamanho da amostra, específica por grupo, considerou-se a variável dependente - complicações respiratórias do RN - com freqüência de $26,0 \%$ entre os não expostos à PTP, o RR de 2,5 , o nivel de confiança de $95,0 \%(\alpha=0,05)$ e o poder estatístico de $90,0 \%(\beta=0,10)$. O tamanho amostral mínimo calculado foi de 121 gestantes portadoras de uma cesárea anterior em cada grupo $^{13}$.

A PTP, considerada como variável independente, foi definida pela presença de contrações uterinas rítmicas, freqüentes e progressivas, espontâneas ou induzidas ${ }^{3}$.

Foram caracterizadas como variáveis dependentes: a via de parto (vaginal ou cesárea) e as complicações puerperais, quando se avaliou a presença ou não de complicações maternas, ocorridas no período periparto e puerperal imediato ou tardio; foram elas: retenção placentária, rotura uterina, realização de histerectomia, celulite de parede, infecção puerperal, óbito materno e outras. Entre as complicações neonatais foram estudados os índices de Apgar - avaliados no primeiro e quinto minutos de vida, relacionados em duas categorias, menor do que $7(<7)$ e maior ou igual a $7(\geq 7)$ - ; o indice de morte perinatal - relacionado à ocorrência de óbito fetal (natimortos) e de óbito neonatal precoce (neomortos até o sétimo dia de vida) - e as complicações respiratórias dos RN relativas ao tipo de parto, caracterizadas por distúrbio respiratório leve e taquipnéia transitória do recém-nascido, considerando-se a presença (SIM) ou ausência (NÃO).

Os dados dos RN foram obtidos dos respectivos prontuários, a partir das avaliações dos neonatologistas, desde a internação até a alta no berçário. Por se tratar de trabalho retrospectivo, não foi possível avaliar a morbimortalidade neonatal após a alta do berçário. Na maioria das vezes, o acompanhamento dos RN não foi realizado no Serviço.

Foram considerados como variáveis de controle alguns fatores, maternos e fetais, relacionados à indicação da PTP. Entre eles, a idade materna em anos completos, por ocasião do parto, agrupada em três faixas etárias, 10 a 19, 20 a 39 e 40 anos ou mais; a paridade anterior - número de partos com feto viável, anterior ao parto estudado, categorizada em primíparas (1 parto) e multíparas ( $\geq 2$ partos); a assistência pré-natal - definida pelo 
número de consultas, referida como SIM ( $\geq 5$ consultas) e NÃO ( $<5$ consultas) e as doenças maternas intercorrentes - presença ou não de doenças clínicas e/ou obstétricas na gestação e no parto em estudo. Entre as doenças clínicas foram relacionadas: cardiopatia, diabete, sindromes hipertensivas, incompetência ístmo-cervical e condilomatose, e entre as obstétricas: hemorragias do terceiro trimestre, trabalho de parto prematuro, rotura prematura de membranas, oligoâmnio, polidrâmnio e outras. Das varáveis relacionadas aos $\mathrm{RN}$, foram incluídas a classificação do RN e a presença (SIM) ou ausência (NÃO) de malformações de qualquer tipo ou etiologia, diagnosticadas na gestação ou ao nascimento. Para a classificação do RN, consideraram-se a idade gestacional e a relação peso/idade ao nascimento, obtendo-se as seguintes categorias: RN pré-termo: idade gestacional inferior a 37 semanas; RN de termo: idade gestacional superior a 37 semanas; RN de peso adequado: peso entre os percentis 10 e 90; RN de peso pequeno: peso abaixo do percentil 10; RN de peso grande: peso acima do percentil 90.

No estudo estatístico foram realizadas análises do tipo uni e multivariada. Para a análise univariada foram obtidas freqüências de todas as variáveis estudadas e montadas as tabelas, relacionando a variável independente com as variáveis dependentes. Na comparação das proporções foi utilizado o teste do qui-quadrado $\left(\chi^{2}\right)$, adotando $5 \%$ como limite de significância $(\mathrm{p}<0,05)^{13}$. Para a análise multivariada, empregou-se a regressão logística, com intervalos de confiança calculados a partir dos coeficientes de regressão e de seus respectivos erros padrões. Essa técnica de análise permitiu estimar o odds ratio em relação à categoria da referência de cada valor ${ }^{14}$.

Este projeto foi aprovado pelo Comitê de Ética em Pesquisa da Faculdade de Medicina de Botucatu - UNESP.

\section{Resultados}

Das 438 gestantes incluídas no estudo, 299 $(68,3 \%)$ foram testadas quanto à PTP, das quais 177 gestantes $(59,2 \%)$ tiveram seu parto por via vaginal. A cesárea eletiva foi realizada em 139 $(31,7 \%)$ dos casos.

Em relação aos fatores maternos a PTP foi mais indicada em gestantes mais jovens ( $<20$ anos) e sem doenças associadas, clinicas ou obstétricas, e contra-indicada nas mais idosas ( $\geq 40$ anos) e naquelas portadoras de sindromes hipertensivas e de hemorragias do terceiro trimestre. A pa- ridade e a assistência pré-natal não se relacionaram à indicação da PTP. Dos fatores perinatais, apenas a prematuridade contra-indicou a PTP. A classificação peso/idade gestacional do RN e a presença de malformações não interferiram com a indicação da PTP (Tabela 1).

Tabela 1 - Fatores maternos e perinatais relacionados à indicação da prova de trabalho de parto (PTP) em gestantes com uma cesárea anterior.

\begin{tabular}{|c|c|c|c|c|c|}
\hline \multirow[t]{3}{*}{ Fatores } & \multicolumn{4}{|c|}{ PTP } & \multirow[t]{3}{*}{$p$} \\
\hline & \multicolumn{2}{|c|}{ Sim } & \multicolumn{2}{|c|}{ Não } & \\
\hline & $\mathrm{n}$ & $\%$ & $\mathrm{n}$ & $\%$ & \\
\hline \multicolumn{6}{|l|}{ Maternos } \\
\hline Idade $<20$ anos & 33 & 11,0 & 5 & 3,6 & $0,008^{*}$ \\
\hline Idade $\geq 40$ anos & 8 & 2,7 & 9 & 6,5 & $0,008^{*}$ \\
\hline Primiparidade & 232 & 77,6 & 109 & 78,4 & 0,864 \\
\hline $\mathrm{APN}^{* *}$ & 285 & 95,3 & 132 & 95,0 & 0,973 \\
\hline Ausência de doença & 157 & 52,5 & 33 & 23,7 & $0,000^{*}$ \\
\hline Síndromes hipertensivas & 21 & 7,0 & 36 & 25,9 & $0,000^{*}$ \\
\hline Hemorragias 30 trimestre & 1 & 0,3 & 5 & 3,6 & $0,001^{*}$ \\
\hline \multicolumn{6}{|l|}{ Perinatais } \\
\hline Prematuridade & 68 & 22,7 & 69 & 45,4 & $0,000^{*}$ \\
\hline Peso pequeno & 22 & 7,4 & 17 & 11,2 & 0,325 \\
\hline Peso grande & 31 & 10,3 & 17 & 11,2 & 0,325 \\
\hline Malformações & 26 & 8,7 & 17 & 11,2 & 0,396 \\
\hline
\end{tabular}

A maioria das grávidas $(89,0 \%)$ não apresentou qualquer intercorrência no parto e puerpério e as complicações observadas não foram relacionadas à PTP. A ocorrência de celulite de parede, infecção puerperal e de óbito materno se distribuiu de forma semelhante entre os grupos. Apesar da pequena proporção, foram complicações exclusivas do grupo de gestantes submetidas à PTP a retenção placentária $(2,0 \%)$, a rotura uterina $(1,0 \%)$ e a realização de histerectomia no pós-parto $(0,3 \%)$ (Tabela 2$)$.

Das 438 gestantes avaliadas, 11 eram portadoras de gestação múltipla - 10 gemelares e uma trigemelar, totalizando 450 conceptos. Nasceram vivos 292 RN no grupo submetido à PTP e 148 entre os de cesárea eletiva. A taxa de óbito fetal variou de 1,3 a $2,7 \%$ e a neomortalidade precoce, entre 2,0 e $4,0 \%$, sem relação com a PTP. A mortalidade perinatal, total ou corrigida pela exclusão das malformações, foi semelhante entre os dois grupos, também, independente da PTP. Os indices de Apgar $<7$ no $1^{\circ}$ e $5^{\circ}$ minutos foram semelhantes entre os dois grupos de mães testa- 
das ou não quanto à PTP, entretanto, as complicações respiratórias ocorreram em menor proporção nos recém-nascidos de mães que realizaram a PTP (Tabela 3).

Tabela 2 - Complicações maternas no parto e puerpério em gestantes com uma cesárea anterior testadas ou não quanto à prova de trabalho de parto (PTP)

\begin{tabular}{|c|c|c|c|c|c|}
\hline \multirow[t]{3}{*}{ Complicações } & \multicolumn{4}{|c|}{ PTP } & \multirow[t]{3}{*}{$\mathrm{p}$} \\
\hline & \multicolumn{2}{|c|}{ Sim } & \multicolumn{2}{|c|}{ Não } & \\
\hline & $\mathrm{n}$ & $\%$ & $\mathrm{n}$ & $\%$ & \\
\hline Ausentes & 267 & 89,3 & 124 & 89,2 & \\
\hline Retenção de placenta & 6 & 2,0 & - & - & \\
\hline Rotura uterina & 3 & 1,0 & - & - & \\
\hline Histerectomia & 1 & 0,3 & - & - & \\
\hline Celulite de parede & 4 & 1,3 & 4 & 2,9 & $0,27 \mathrm{~ns}$ \\
\hline Infecção puerperal & 3 & 1,0 & 3 & 2,2 & $0,34 \mathrm{~ns}$ \\
\hline Óbito & 1 & 0,3 & 2 & 1,4 & $0,24 \mathrm{~ns}$ \\
\hline Outras & 14 & 4,8 & 6 & 4,3 & \\
\hline
\end{tabular}

ns: diferença não significativa $(p>0,05)$.

Tabela 3 - Total de recém-nascidos vivos, índices de morte perinatal (MPN) e de Apgar $<7$ e ocorrência de complicações respiratórias nos recém-nascidos de gestantes com uma cesárea anterior testadas ou não quanto à prova de trabalho de parto (PTP).

\begin{tabular}{lrrrrr}
\hline Recém-nascidos & \multicolumn{3}{c}{ PTP } & P \\
& \multicolumn{1}{c}{ Sim } & \multicolumn{2}{c}{ Não } & \\
& n & \multicolumn{1}{c}{$\mathbf{n}$} & \multicolumn{1}{c}{$\%$} & \\
\hline Nativivos & 292 & 97,3 & 148 & 98,7 & \\
Natimortos & 8 & 2,7 & 2 & 1,3 & \\
Neomortos & 6 & 2,0 & 6 & 4,0 & \\
MPN Total (\%) & & 46,7 & & 53,3 & 0,147 \\
$\quad$ Corrigida** & & 20,0 & & 40,0 & \\
Apgar <7 - 10 minuto & 42 & 14,4 & 23 & 15,8 & 0,791 \\
Apgar <7 - 50 minuto & 12 & 4,1 & 8 & 5,5 & 0,698 \\
Complicações respiratórias & 9 & 3,1 & 11 & 7,4 & 0,010 \\
\hline
\end{tabular}

* = diferença significativa $(p<0,05)$.

** = excluídas as malformações.

Na análise multivariada, a indicação da PTP dependeu da idade materna e da ausência de doença associada: a idade materna mais avançada contra-indicou a prova, e a ausência de doenças clínicas e/ou obstétricas aumentou em mais de três vezes a indicação da PTP. As complicações maternas não dependeram da PTP; o término da gestação por cesárea triplicou a ocorrência de complicações no parto e puerpério. A mortalidade perinatal e os índices de Apgar $<7$ guardaram relação inversa com o peso do RN: quanto maior o peso do RN, menor o risco de morte perinatal e de Apgar $<7$. Este índice também dependeu da pre- sença de malformações: a presença de malformações implicou maior risco $(2,5$ vezes) de índices de Apgar $<7$. As complicações respiratórias do RN foram quase duas vezes mais freqüentes nos RN de mães que não realizaram a PTP. Estes resultados da análise multivariada, estatisticamente significativos, estão representados na Figura 1.

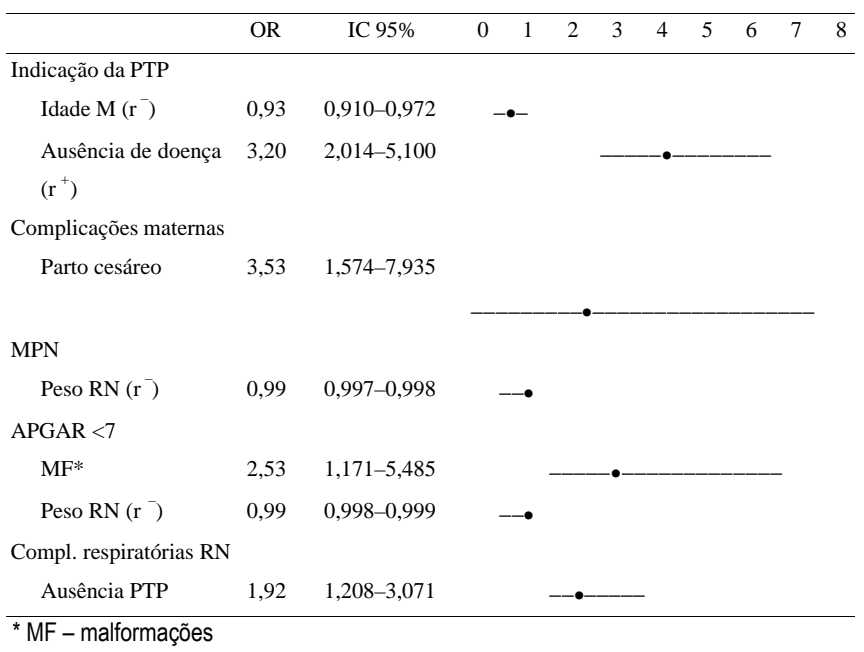

Figura 1 - Representação gráfica das variáveis significativas evidenciadas na análise multivariada - odds ratio (OR) e intervalo de confiança (IC) a 95\% - relacionadas à indicação da prova de trabalho de parto (PTP), às complicações maternas, à mortalidade perinatal (MPN), aos índices de Apgar e às complicações respiratórias dos recém-nascidos (RN).

\section{Discussão}

A PTP realizada em gestantes com uma cesárea anterior levou à ocorrência de $59,2 \%$ de partos vaginais. Na literatura o índice de sucesso da PTP varia de 60 a 90\% $3,9,10,15$, dependendo do tipo de estudo. A meta-análise desenvolvida por Rosen et al. ${ }^{9}$ evidenciou índices entre 39 e $89 \%$; Flamm et al. ${ }^{10}$, em estudo multicêntrico, encontraram $75 \%$ e Lao e Leung ${ }^{15}$, em revisão de 137 pacientes com PTP induzida por drogas, verificaram $81,8 \%$ de partos vaginais após uma cesárea anterior.

A indicação da PTP não dependeu da paridade e da qualidade da assistência pré-natal, mas foi relacionada à idade materna. As mulheres com idade igual ou superior a 40 anos foram menos submetidas à prova, ao passo que nas adolescentes investiu-se mais na tentativa da via vaginal. Cnattingius et al. ${ }^{16}$, em estudo retrospectivo, confirmaram risco aumentado de cesárea em portadoras de uma cesárea anterior e idade superior a 35 anos. Parece colaborar para isso a ocorrência de doenças clínicas e obstétricas, mais comuns em gestantes idosas ${ }^{3}$. As sindromes hipertensivas e as hemorragias do $3^{\circ}$ trimestre interferiram de modo desfavorável na indicação da PTP, evidenciado pela maior proporção de cesárea 
eletiva. Em gestantes sem cicatriz prévia de cesárea as sindromes hipertensivas e as hemorragias do final da gestação são apontadas como indicações relativas do parto por via abdominal. A placenta prévia é contra-indicação à realização da $\mathrm{PTP}^{17}$ e as síndromes hipertensivas, pela gravidade dos casos atendidos num hospital terciário, se associam a altos índices de cesárea. Quanto às características dos RN, o único fator que interferiu na decisão de realizar ou não a PTP foi a idade gestacional - nos prematuros se investiu pouco na obtenção do parto vaginal, fato que não foi confirmado na análise de Pires ${ }^{12}$. Os resultados ressaltaram as condições ideais para a PTP em gestantes com uma cesárea anterior já identificadas por outros autores ${ }^{3,4,10,11}$. Entretanto, a falta dessas condições não deve servir para contra-indicar a prova, uma vez que os indicadores de sucesso ainda não estão completamente definidos ${ }^{3,18,19}$. Ao contrário, as contra-indicações, já estabelecidas, devem sempre ser respeitadas ${ }^{3,9-11}$.

As complicações maternas não foram relacionadas à PTP, atingindo cerca de $10,0 \%$ das pacientes em ambos os grupos. Os três casos de rotura uterina observados no grupo de gestantes submetidas à PTP evoluíram satisfatoriamente, necessitando apenas de histerorrafia. Em dois deles administrou-se ocitocina para indução ou condução do trabalho de parto, o que contribuiria para um pior prognóstico. Segundo alguns autores, são fatores de pior prognóstico nesses casos a rotura completa, com expulsão do feto na cavidade abdominal, e o maior tempo decorrido entre o diagnóstico e a intervenção $0^{3,20}$. Além disso, a associação entre rotura completa, uso de ocitocina e falha da PTP já foi descrita por outros autores, comprometendo o prognóstico materno e fetal ${ }^{3,21}$. O único caso de histerectomia puerperal ocorreu por acretismo placentário e não se relacionou à PTP ou à rotura uterina.

A mortalidade materna em gestantes com cesárea anterior é baixa, mas não é nula, e por isso deve ser valorizada ${ }^{3}$. Em geral são descritos em cada estudo um ou dois casos relacionados à infecção pós-parto, ao acretismo placentário e à falha da PTP, culminando em cesárea de urgência $^{3,9-11}$. Neste trabalho, dois dos três óbitos maternos aconteceram no grupo com cesárea eletiva, ambos diretamente relacionados às síndromes hipertensivas - eclâmpsia e sindrome HELLP. O terceiro óbito foi de uma gestante com rotura prematura de membranas, submetida à PTP sem sucesso, que desenvolveu infecção pós-parto.

O índice de Apgar $<7$ não mostrou relação com a PTP, mas sim com a presença de malformações e com o peso dos RN. O maior peso dos RN e a ausência de malformações diminuíram o risco de Apgar <7 e, conseqüentemente, a ocorrên- cia de depressão neonatal, que acometeu cerca de $15 \%$ dos neonatos, sem relação com a PTP. Ao contrário, os distúrbios respiratórios se manifestaram em maior proporção nos RN de mães com cesárea eletiva, reafirmando que as complicações respiratórias são comuns na cesárea eletiva ${ }^{22}$. O fato de não realizar a PTP aumentou em quase duas vezes o risco de complicações respiratórias do RN, resultado esperado e justificado, que reforça a validade dessa estratégia.

Nenhum óbito perinatal aconteceu no período intraparto. A análise de Meehan et al. ${ }^{22}$, no final da década de 80 , salientou o risco de elevação da mortalidade perinatal com a realização de PTP em gestantes com cesárea anterior. Uma década mais tarde, outros autores, comparando gestantes testadas quanto à PTP e submetidas à cesárea eletiva, comprovaram não haver diferença significativa nestes índices ${ }^{5}$.

Em geral, a mortalidade perinatal em gestantes com cesárea anterior é de $5,0 \%$ o nascimentos $^{9}$. Aqui este coeficiente foi bem mais elevado, apesar de semelhante entre os grupos. As características do Serviço, onde importante contingente de baixo peso, malformados e prematuros é rotina, poderiam explicar esse achado. Entretanto, a análise multivariada mostrou que o menor peso do $\mathrm{RN}$ foi o fator determinante da mortalidade perinatal e não se relacionou à realização da PTP.

Apesar de tendência atual em se observar maior número de mulheres com uma cesárea anterior e o parto consecutivo por via vaginal, esta realidade está longe de ser atingida no Brasil. Fatores médicos estão claramente relacionados e algumas intervenções específicas talvez diminuam os índices epidêmicos das cesáreas de repetição ${ }^{20}$. A indicação precisa da primeira cesárea, ponderando não só os riscos diretos como também os potenciais de seqüelas tardias para a mãe e o recém-nascido ${ }^{22}$, deve ser uma das estratégias.

Considerando que nova cesárea foi evitada em $59,2 \%$ das gestantes testadas quanto à PTP e que a morbimortalidade, materna e perinatal, não se relacionou à sua realização ou à possibilidade de falha e conseqüente resolução por cesárea, esta estratégia deve ser estimulada em gestantes com uma cesárea anterior.

\section{ABSTRACT}

Purpose: to study trial of labor (TOL) for vaginal birth after one previous cesarean section.

Methods: this is a retrospective cohort study that included 438 pregnant women with one previous cesarean section and their 450 newborns. They were divided into two groups 
- with and without TOL. The minimum sample size was 121 pregnant mothers per group. TOL was considered as an independent variable and vaginal birth and maternal and perinatal complication frequency as dependent variables. Both univariate and multivariate analyses were performed. The comparison of observed frequencies (\%) was analyzed by the chi-squared test $\left(\chi^{2}\right)$ with $5 \%$ significance, and linear regression from the odds ratio (OR) and confidence interval of $95 \%\left(\mathrm{CI}_{95 \%}\right)$.

Results: TOL was used in 59.2\% of vaginal deliveries. It was less used in women over 40 years (2.7\% vs 6.7\%) and in those with clinical or obstetrical diseases such as arterial hypertension (7.0\%) and bleeding in the third trimester $(0.3 \%)$. There was a higher risk for puerperal complications with cesarean deliveries $(O R=3.53$, CI 95\% = 1.57-7.93), independent of TOL. Perinatal mortality was dependent on neonatal weight and fetal malformations, not on TOL. Newborns from mothers not submitted to TOL were at a higher risk for developing breathing complications $(\mathrm{OR}=1.92 \mathrm{CI}$ $95 \%=1.20-3.07$ ).

Conclusions: The results confirm that trial of labor after a previous cesarean section is a safe method-assisting vaginal delivery in 59.2\% of births and not interfering with maternal and perinatal mortality. It is a treatment that should be stimulated.

KEY WORDS: Trial of labor. Previous cesarean section. Maternal and perinatal complications.

\section{Agradecimentos}

Ao Conselho Nacional de Pesquisa e Desenvolvimento pela concessão da bolsa de Iniciação Cientifica.

\section{Referências}

1. Sachs BP, Yeh J, Acker D, Driscoll S, Brown DA, Jewett JF. Cesarean section-related maternal mortality in Massachusetts, 1954-1985. Obstet Gynecol 1988; 71:385-8.

2. Faúndes A, Ceccati JG. Which policy for cesarean sections in Brazil? An analysis of trends and consequences. Health Policy Plan 1993; 8:33-42.

3. McMahon MJ. Vaginal birth after cesarean. Clin Obstet Gynecol 1998; 41:369-81.

4. ACOG Committee Opinion. Committee on Obstetrics. Maternal and fetal medicine guidelines for vaginal delivery after a previous cesarean section. Washington, DC: American College of Obstetricians and Gynecologists; 1998. (Bulletin $n^{\circ}$ 64).

5. Rageth JC, Juzi C, Grossenbacher H. Delivery after previous cesarean: a risk evaluation. Obstet Gynecol 1999; 93:332-7.

6. Rosen MG, Dickinson JC. Vaginal birth after cesarean: a meta-analysis of indicators for success. Obstet Gynecol 1990; 76:865-9.
7. Flamm BL, Newman LA, Thomas SJ, Fallon D, Yoshida MM. Vaginal birth after cesarean delivery: results of a 5-year multicenter collaborative study. Obstet Gynecol 1990; 76:750-4.

8. McMahon MJ, Luther ER, Bowes WA Jr, Olshan AF. Comparison of a trial of labor with an elective second cesarean section. N Engl J Med 1996; 335:689-95.

9. Rosen MG, Dickinson JC, Westhoff CL. Vaginal birth after cesarean: a meta-analysis of morbidity and mortality. Obstet Gynecol 1991; 77:465-70.

10.Flamm BL, Lim OW, Jones C, Fallon D, Newman LA, Mantis JK. Vaginal birth after cesarean section: results of a multicenter study. Am J Obstet Gynecol 1988; 158:1079-84.

11.Nguyen TV, Dinh TV, Suresh MS, Kinch RA, Anderson GD. Vaginal birth after cesarean section at the University of Texas. J Reprod Med 1992; 37:880-2.

12.Pires HMB. Fatores associados à prova de trabalho de parto e ao parto vaginal em gestantes com uma cesárea anterior [dissertação]. Campinas: Universidade de Campinas; 1996.

13.Fletgher RH, Fletcher SW, Wagner EH. Epidemiologia clinica: elementos essenciais. $3^{\mathrm{a}}$ ed. Porto Alegre: Artes Médicas; 1996. p.281.

14.Hosmer DW, Lemeshow S. Applied logistic regression. $1^{\text {st }}$ ed. New York: John Wiley \& Sons; 1989. p.307.

15.Lao TT, Leung BF. Labor induction for planned vaginal delivery in patients with previous cesarean section. Acta Obstet Gynecol Scand 1987; 66:413-6.

16.Cnattingius R, Cnattingius S, Notzon FC. Obstacles to reducing cesarean rates in a low-cesarean setting: the effect of maternal age, height and weight. Obstet Gynecol 1998; 92:501-6.

17.McMahon MJ, Li R, Schenck AP, Olshan AF, Royce RA. Previous cesarean birth: a risk factor for placenta previa? J Reprod Med 1997; 42:409-12.

18.Troyer LR, Parise VM. Obstetric parameters affecting success in a trial of labor: designation of a scoring system. Am J Obstet Gynecol 1992; 167:1099-104.

19.Pickhardt MG, Martin JN Jr, Meydrech EF, et al. Vaginal birth after cesarean delivery: are there useful and valid predictors of success or failure? Am J Obstet Gynecol 1992; 166:1811-9.

20.Cecatti JG, Andreucci CB, Cacheira PS, Pires HMB, Pinto e Silva JL, Aquino MMA. Fatores associados à realização de cesárea em primíparas com uma cesárea anterior. Rev Bras Ginecol Obstet 2000; 22:175-9.

21.Farmer RM, Kirschbaum T, Potter D, Strong TH, Medearis AL. Uterine rupture during trial of labor after previous cesarean section. Am J Obstet Gynecol 1991; 165:996-1001.

22.Meehan FP, Burke G, Casey C, Sheil JG. Delivery following cesarean section and perinatal mortality. Am J Perinatol 1989; 6:90-4.

Recebido em: 27/04/01 Aceito com modificações em: 28/03/02 